\title{
Inhibiting PSMa-induced neutrophil necroptosis protects mice with MRSA pneumonia by blocking the agr system
}

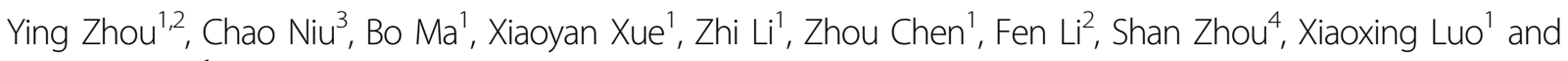
Zheng Hou (1)

\begin{abstract}
Given its high resistance, enhanced virulence, and high transmissibility, community-associated methicillin-resistant Staphylococcus aureus (CA-MRSA) pneumonia is highly associated with high morbidity and mortality. Anti-virulence therapy is a promising strategy that bypasses the evolutionary pressure on the bacterium to develop resistance. RNAlllinhibiting peptide (RIP), as an accessory gene regulator (agr)-specific inhibitor, significantly restricts the virulence of $S$. aureus and protects infected mice from death by blocking the agr quorum sensing system. The protective effects of RIP on the neutropenic mice completely disappeared in a neutrophil-deleted mouse infection model, but not in the macrophage-deleted mice. This result confirmed that the in vivo antibacterial activity of RIP is highly associated with neutrophil function. Phenol-soluble modulins (PSMs), as major leukocyte lysis toxins of CA-MRSA, are directly regulated by the agr system. In this experiment, PSMa1, 2, and 3 significantly induced neutrophil necroptosis by activating mixed lineage kinase-like protein (MLKL) phosphorylation and increasing lactate dehydrogenase release. The $S$. aureus supernatants harvested from the agr or psma mutant strains both decreased the phosphorylation level of MLKL and cell lysis. PSMa1-mediated neutrophil lysis was significantly inhibited by necrosulfonamide, necrostatin-1, TNFa antibody, and WRW4. These results showed PSMa1 induced necroptosis depends on formylpeptide receptor 2 (FPR2)mediated autocrine TNFa. Moreover, the neutrophil necroptosis induced by $S$. aureus was significantly suppressed and pneumonia was effectively prevented by the blockage of agrA and psma expression levels. These findings indicate that PSMa-induced necroptosis is a major cause of lung pathology in S. aureus pneumonia and suggest that interfering with the agr quorum sensing signaling pathway is a potential therapeutic strategy.
\end{abstract}

\section{Introduction}

Staphylococcus aureus (S. aureus) is an important bacterial pathogen that causes various respiratory tract infections in both adult and pediatric populations ${ }^{1}$. To date, methicillin-resistant $S$. aureus (MRSA) has instigated an antibiotic resistance crisis to commonly used

\footnotetext{
Correspondence: Xiaoxing Luo (xxluo3@fmmu.edu.cn) or Zheng Hou (hzh_0001@163.com)

${ }^{1}$ Department of Pharmacology, School of Pharmacy, Fourth Military Medical University, Xi'an, China

²Department of Pharmacology, Xi'an Medical University, Xi'an, China Full list of author information is available at the end of the article These authors contributed equally: Ying Zhou, Chao Niu, Bo Ma. Edited by A. Oberst.
}

antibiotics in the clinics due to overuse. Linezolid and vancomycin are the most reliable therapeutic agents against MRSA pneumonia ${ }^{2,}{ }^{3}$; however, vancomycinresistant S. aureus (VRSA) and linezolid-resistant S. aureus (LRSA) have alarmingly emerged in severe MRSA pneumonia cases $^{4,}{ }^{5}$. Moreover, the development of antibiotic resistance is associated with high morbidity and mortality risk, particularly in the intensive care unit ${ }^{6}$. Therefore, novel strategies are urgently needed for treating and preventing invasive MRSA infections.

Quorum sensing is a bacterial intercellular communication mechanism that controls the pathogenesis of 
many organisms by regulating gene expression. This mechanism targets many virulence strategies and components, such as virulence determinants, biofilm formation, and drug resistance ${ }^{7}$. The quorum sensing system has become an attractive target for the development of novel anti-infective agents and helps reduce the potential development of bacterial resistance ${ }^{8,9}$. In staphylococci, the heptapeptide RNAIII-inhibiting peptide (RIP) can inhibit the activation of the accessory gene regulator (agr) quorum sensing system and decrease staphylococcal virulence by interfering with the phosphorylation of TRAP (target of RNAIII-activating peptide) ${ }^{8,10}$.

Phenol-soluble modulins (PSMs) are recently discovered amphipathic alpha-helical peptides that are mainly expressed by highly virulent $S$. aureus and are closely related to staphylococcal pathogenesis. The enhancement of PSMs production is strictly and directly promoted by the agr quorum sensing regulation system at high cell densities ${ }^{11}$. PSMs are produced at considerable amounts in community-associated MRSA (CA-MRSA), such as LAC (USA300), but are expressed at lower amounts in hospital-associated MRSA (HA-MRSA) ${ }^{12,13}$. HA-MRSA with overexpressed $\alpha$-type PSMs causes neutrophil lysis activity similar to that caused by CA-MRSA strains $^{14,15}$. Therefore, $\alpha$-type PSMs are mainly responsible for the pronounced in vivo leukocidal activity in addition to chemotactic and proinflammatory activities ${ }^{12,}$ ${ }^{13}$. Moreover, $\alpha$-type PSMs have been demonstrated to dramatically influence the infectivity of CA-MRSA in mouse bacteremia, skin abscess, and peritonitis models ${ }^{13}$, 14,16 .

Neutrophil, as an important host innate immune cell, plays a key role in staphylococcal infections by phagocytosis. However, $15-50 \%$ of the initial ingested S. aureus survives within the neutrophil phagosome and likely contributes to disease pathogenesis. In 2014, GreenleeWacker et al. first reported on the intraphagosomal $S$. aureus that induces programmed necrosis in neutrophils ${ }^{17}$. This programmed necrosis further exacerbates staphylococcal disease by releasing viable $S$. aureus and neutrophil constituents ${ }^{17}$. Viable $S$. aureus can propagate infection to distant sites, and neutrophil constituents may cause substantial tissue damage ${ }^{18,19}$.

Based on the evidence from these studies, we hypothesized that the agr blocking strategy may inhibit neutrophil necroptosis by reducing the critically virulent PSMa release and promoting S. aureus clearance. So we investigated the possible anti-infective mechanisms of RIP as an agr system inhibitor in this experiment. Data showed that PSM $\alpha$-induced neutrophil necroptosis was blocked, LAC elimination was enhanced in the pneumonia mouse model, tissue damage was alleviated, and the mouse survival rate was greatly improved by interfering with the agr quorum sensing system.

\section{Results}

Bacterial growth was not inhibited by RIP in vitro

Six Staphylococcus strains were cultured in MuellerHinton $(\mathrm{MH})$ Broth medium in the absence or presence of RIP at different concentrations. MIC assay results showed that RIP did not exert bactericidal effects at $256 \mu \mathrm{g} / \mathrm{mL}$ in vitro (Table 1 ). The growth curve demonstrated that RIP did not inhibit the growths of the six strains in vitro at concentrations up to $1000 \mu \mathrm{g} / \mathrm{mL}$. Meanwhile, vancomycin was used as positive control, which completely inhibited the growths of the six tested strains at $64 \mu \mathrm{g} / \mathrm{mL}$ (Supplementary Figure 1).

\section{MRSA-infected mice were protected by blocking the agr system}

RIP significantly accelerated the body weight recovery of the LAC-infected mice relative to that of the model group (Fig. 1a). Administering $20 \mathrm{mg} / \mathrm{kg}$ RIP to the LACinfected group significantly improved the animal survival rate from $33.3 \%$ for the control groups to $83.3 \%$ (Fig. 1b). Survival was associated with reduced bacterial titers in the lung and bronchoalveolar lavage fluid (BALF) of the infected mice (Fig. 1c, d). To assess the pathological changes, we performed hematoxylin-eosin (HE) staining, measured the lung wet/dry weights, and applied a lung injury score system in our study. Results showed that RIP can significantly reduce pulmonary edema and improve the lung injury score (Fig. 1e-g).

\section{agrA, psma, and psm $\beta$ expression levels were inhibited by blocking the agr system}

Virulence factors of PSMs are regulated by agrA in $S$. aureus. RIP reduced the expression of agrA dosedependently (Fig. 2a, b). Moreover, expression levels of agrA-dependent virulence factors $p s m \alpha$ and $p s m \beta$ were decreased (Fig. 2c, d).

Table 1 MICs of RIP and five antibiotics on six Staphylococcus strains in MH broth culture

\begin{tabular}{lllllll}
\hline \multirow{2}{*}{ Strains } & \multicolumn{7}{l}{ MIC $(\boldsymbol{\mu} \mathbf{g} / \mathbf{m L})$} & & & \\
\cline { 2 - 7 } & RIP & VAN & OXA & CIP & CAZ & LVX \\
\hline S. aureus ATCC29213 & $>256$ & 0.5 & 0.25 & $\leq 0.25$ & 8 & $\leq 0.25$ \\
LAC (USA300) & $>256$ & 0.5 & 2 & 8 & 256 & 4 \\
Mu50 & $>256$ & 8 & 16 & 32 & 256 & 8 \\
MRSA XJ75302 & $>256$ & 0.5 & $>256$ & 16 & 256 & 8 \\
S. epidermidis ATCC14990 & $>256$ & 0.5 & 0.125 & 0.125 & 16 & 0.125 \\
MRSE XJ75284 & $>256$ & 1 & $>256$ & 8 & 256 & 8 \\
\hline
\end{tabular}

VAN vancomycin, OXA oxacillin, CIP ciprofloxacin, CAZ ceftazidine, LVX levofloxacin 

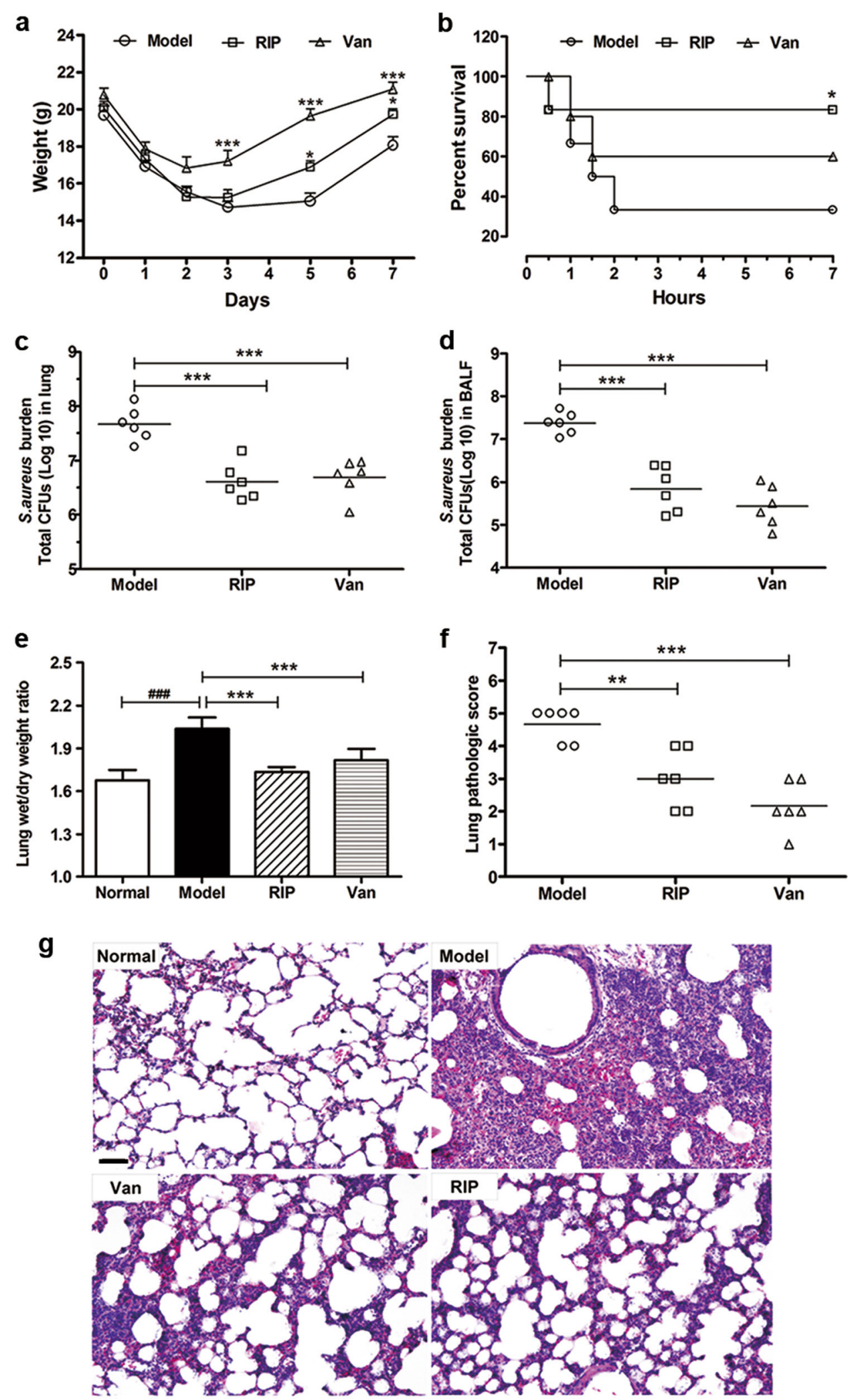

Fig. 1 (See legend on next page.) 
Fig. 1 LAC-infected pneumonia mice were protected by blocking the agr system. a The body weight of LAC-infected mice recovered after treated by $20 \mathrm{mg} / \mathrm{kg}$ RIP or $10 \mathrm{mg} / \mathrm{kg}$ vancomycin $(n=7)$. b Survival of BALB/c mice nasally inoculated with $L A C\left(3 \times 10^{7} \mathrm{CFU}\right)$ and treated with PBS, RIP, or vancomycin by i.p. injection at 1 and $6 \mathrm{~h}$ after infection $(n=12)$. $\mathbf{c}$, $\mathbf{d}$ The number of CFU in the lung (c) or in the BALF (d) was calculated from the number of colonies growing on plates $(n=6)$. e Effects of RIP on lung injury in LAC-infected mice were analyzed by Lung wet/dry weight $(n=6)$. f After $24 \mathrm{~h}$ infection, the left lung of infected mice were harvested, fixed, stained with $\mathrm{HE}$, and analyzed microscopically. The total pathologic score for each mouse was calculated. Severity was graded on a scale of $0-5$, with 0 representing normal lung and 5 representing severe pneumonia $(n=6)$. g Lung histopathologic examination of LAC-induced pneumonia $24 \mathrm{~h}$ after bacterial challenge in mice. The original magnification is $\times 200$. The bar length represents $50 \mathrm{\mu m}$. Data were shown as mean $(\mathbf{c}, \mathbf{d}, \mathbf{f})$, mean $\pm \mathrm{SD}(\mathbf{e})$, or mean $\pm \mathrm{SE}(\mathbf{a}){ }^{*} P<0.05,{ }^{* *} P<0.01,{ }^{* * *} P<0.0001 \mathrm{vs}$. model group; ${ }^{\# \# \#} P$ $<0.0001$ vs. normal group
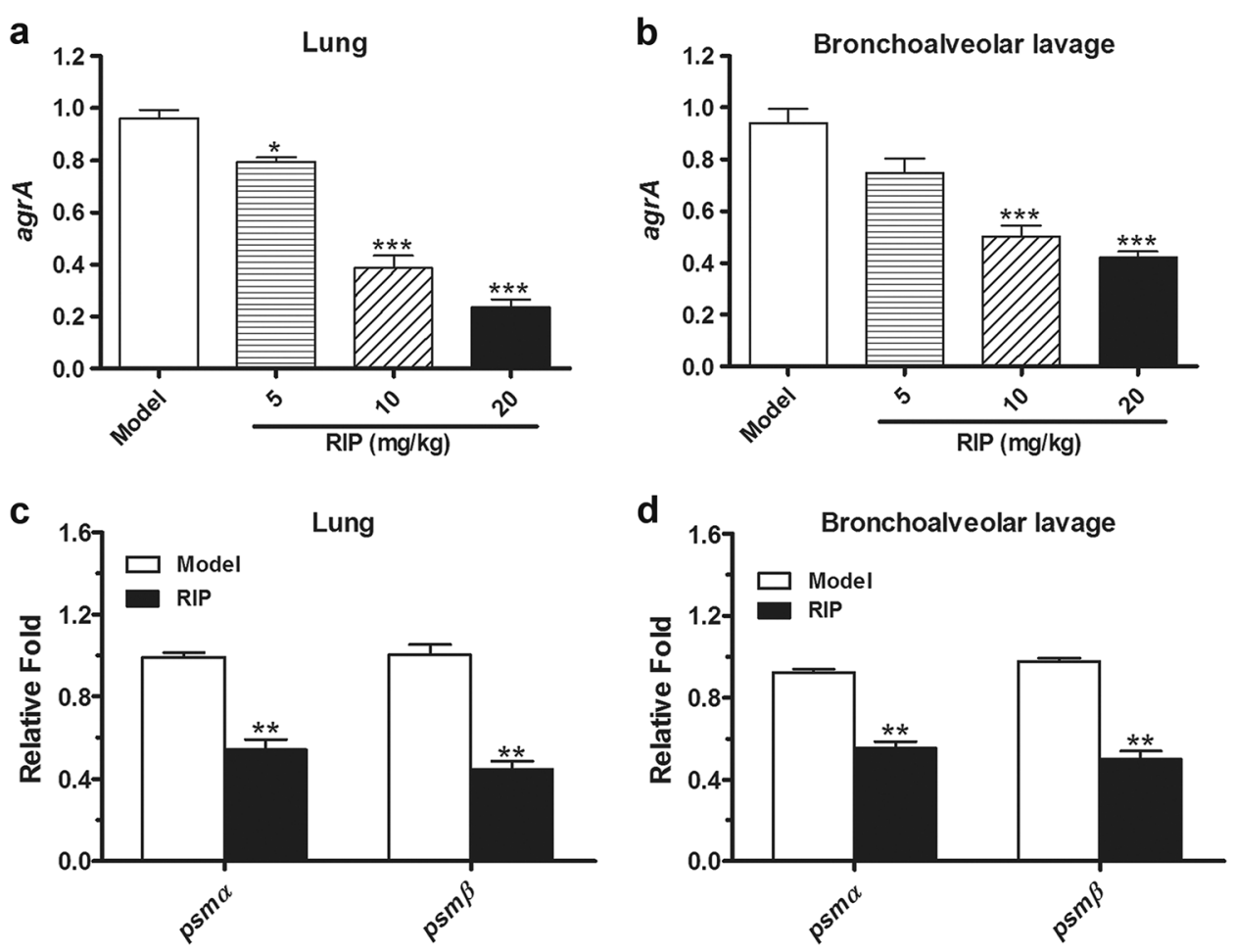

Fig. 2 The quantitative RT-PCR analysis of the agrA, psma, and psm $\beta$ genes in LAC isolated from infected mice. $\mathbf{a}, \mathbf{b}$ The mRNA expression levels of agrA in LAC that isolated from lung tissue or bronchoalveolar lavage fluid in LAC-infected mice following treatment with or without RIP. $\mathbf{c}, \mathbf{d}$ The mRNA expression levels of $p s m a$ and $p s m \beta$ in LAC that isolated from lung tissue or bronchoalveolar lavage fluid. The mRNA levels were quantified after comparison with an internal gene (16S rRNA). Data are expressed as the mean \pm SE $(n=3)$. ${ }^{*} P<0.05,{ }^{* *} P<0.01,{ }^{* * *} P<0.0001$ vs. model group

Neutrophil is essential to RIP's in vivo protective effect

After a single tail-vein injection of $150 \mu \mathrm{L}$ clodronate liposomes or $200 \mathrm{mg} / \mathrm{kg}$ CTX, most neutrophils or macrophages were both depleted in mice at $24,48,96$, and $120 \mathrm{~h}$ (Fig. 3b, d), and CTX had no effect on macrophages in mice peritoneal lavage (Fig. 3f). RIP can significantly improve the survival rate of LAC-infected mice from $18.8 \%$ for the model groups to $86.7 \%$ (Fig. 3a). RIP also exerted a protective effect after the deletion of macrophages with $41.7 \%$ survival rate (Fig. 3c). However, the protective effects of RIP on the neutropenic mice were completely lost, and all the mice died within 2 days in the model and RIP-treated groups (Fig. 3e). Compared with the control group, the $10,30,100,300$, and $1000 \mu \mathrm{g} / \mathrm{mL}$ concentrations of RIP did not affect the myeloperoxidase (MPO) activity of neutrophils, whereas LPS significantly increased the MPO activity of the neutrophils (Fig. 3g). Meanwhile, RIP did not affect the neutrophils' viability and LDH release compared with the control group (Fig. 3h, i). Thus, the RIP did not directly activate the neutrophils.

\section{LAC supernatant induces neutrophil necroptosis}

A S. aureus and neutrophil co-culture system was utilized to investigate the possible mechanisms underlying the antimicrobial effects of the $a g r$ inhibitor. The level of phosphorylation-mixed lineage kinase-like protein (pMLKL) was increased in human neutrophils regardless 

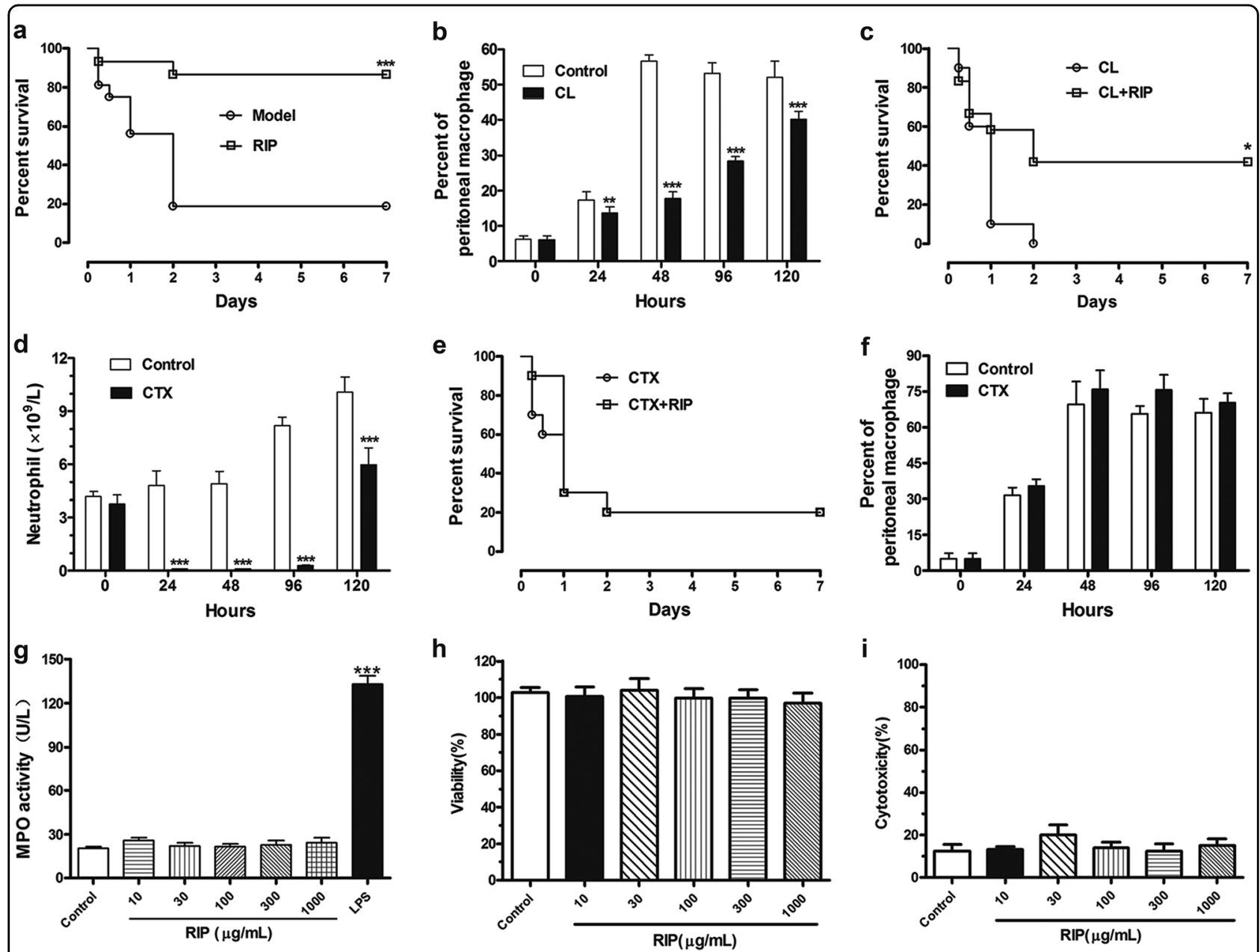

Fig. 3 Neutrophil is essential to RIP's in vivo protective effect. a Survival of LAC-infected mice treated with $20 \mathrm{mg} / \mathrm{kg} \mathrm{RIP}(n=15)$. The model group was given the same volume of PBS $(n=16)$. $\mathbf{b}, \mathbf{f}$ The proportion of macrophages in the peritoneal lavage fluid of mice. BALB/c mice were injected intravenously with $150 \mu \mathrm{L}$ clodronate liposomes $(\mathrm{CL})$ and $200 \mathrm{mg} / \mathrm{kg}$ cyclophosphamide (CTX), and subsequently challenged with $3 \times 10^{7}$ CFU LAC. The proportion of macrophages was analyzed by flow cytometry $(n=3)$. c Survival rate of the macrophage-deleted mice infected with LAC and then treated by $20 \mathrm{mg} / \mathrm{kg}$ of RIP at 1 and $6 \mathrm{~h}$ after infection $(n=10)$. $\mathbf{d}$ Neutrophil depletion was performed in BALB/c mice via administration of $200 \mathrm{mg} / \mathrm{kg}$ CTX, and then the mice were challenged by $3 \times 10^{7}$ CFU LAC. The number of neutrophil in the blood of mice was analyzed with an Abbott Cell-Dyn 3700 system $(n=3)$. e Survival rate of the neutrophils-deleted mice infected with LAC and then treated with $20 \mathrm{mg} / \mathrm{kg}$ RIP at 1 and $6 \mathrm{~h}$ after infection $(n=10)$. g Neutrophils were incubated with $10,30,100,300$, and $1000 \mu \mathrm{g} / \mathrm{mL} \mathrm{RIP} \mathrm{or} 50 \mathrm{ng} / \mathrm{mL}$ LPS alone for 30 min at $37^{\circ} \mathrm{C}$. MPO enzyme activity was measured spectrophotometrically in the cell-free supernatants $(n=4)$. $\mathbf{h}, \mathbf{i}$ After human neutrophils were pretreated with various concentrations of RIP for $24 \mathrm{~h}$ at $37^{\circ} \mathrm{C}$ and $5 \% \mathrm{CO}_{2}$, cell proliferation was measured by CCK-8 $(n=8)$ and the release of LDH amount was determined $(n=6)$. Data were shown as mean $\pm \mathrm{SD}(\mathbf{b}, \mathbf{d})$, or mean $\pm \mathrm{SE}(\mathbf{f}-\mathbf{i}) .{ }^{* *} P<0.01,{ }^{* * *} P<0.0001$ vs. control group

of the LAC or LAC supernatant co-incubation of the neutrophils (Fig. 4a, b). Moreover, the levels of pMLKL and cell lysis were prevented by the MLKL inhibitor Necrosulfonamide (NSA) or receptor-interacting protein kinase 1 (RIPK1) inhibitor Necrostatin-1 (Nec) in a concentration-dependent manner (Fig. 4c-f). The abovementioned data indicated that the $S$. aureus culture supernatant may induce neutrophil necroptosis.

\section{PSMa1 induces neutrophil necroptosis}

To investigate the roles of these PSMs in inducing neutrophil necroptosis, we incubated these seven synthetic peptides (Supplementary Table 1) with neutrophils. Results showed that PSM $\alpha 1,2$, and 3 can increase the level of pMLKL and the release of LDH in neutrophils, which can be significantly inhibited by NSA (Fig. 5a, b). The morphology of neutrophils was observed under transmission electron microscopy (TEM). After treatment with PSM $\alpha 1$, the cell volume increased, the surface morphology became irregular, and the organelles and cell submicrons disappeared (Fig. 5c). However, the morphology and structure of the neutrophils resembled those of the control neutrophil cells after NSA pretreatment (Fig. 5c). Cytotoxicity results showed that MLKL 

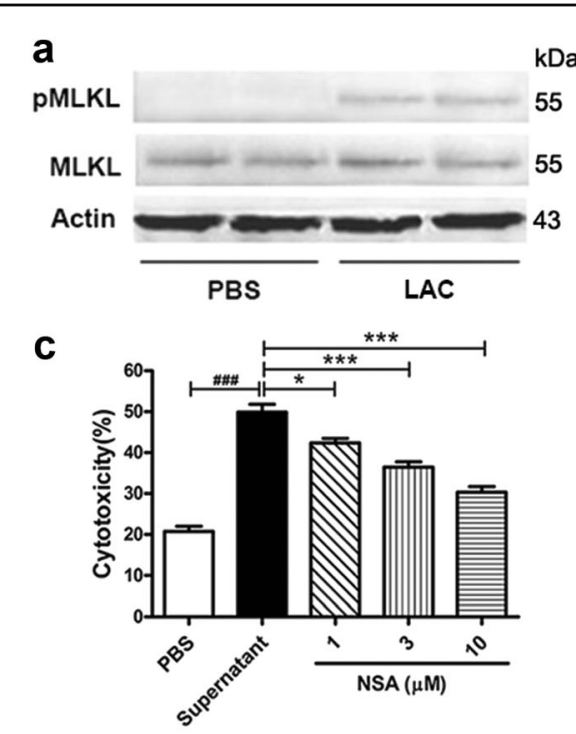

d

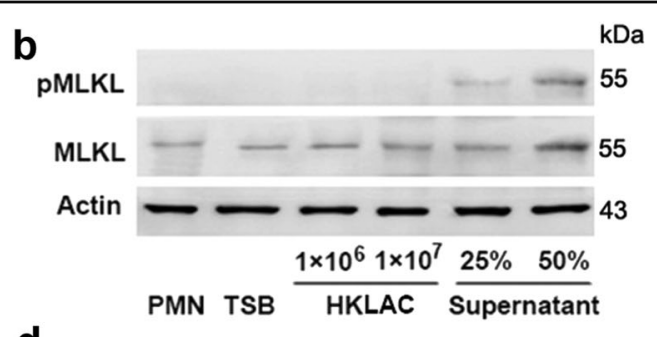

e
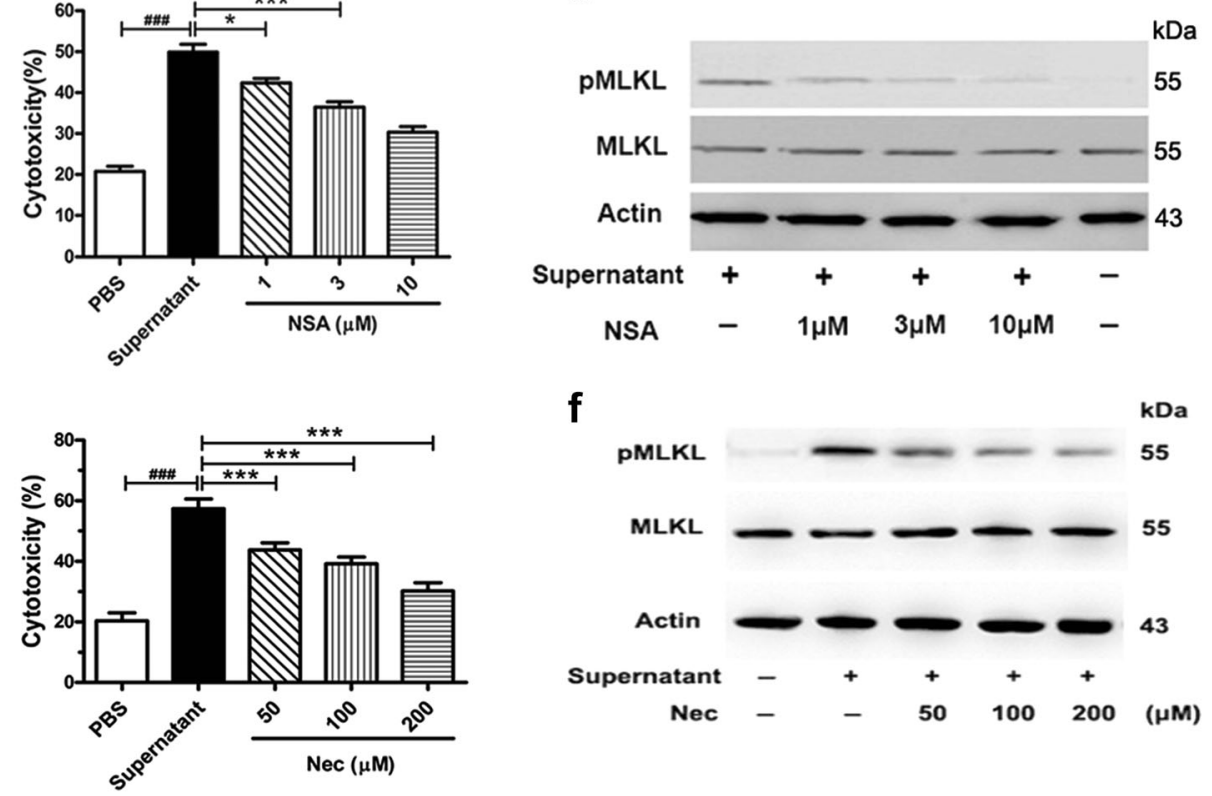

Fig. 4 LAC induced necroptosis in neutrophil. a, b Human neutrophils were stimulated with PBS, LAC (MOI 10), LAC culture supernatant, or HKLAC. Level of phosphorylated MLKL (pMLKL), MLKL, and $\beta$-actin in neutrophil lysate were detected by western blot. $\mathbf{c}$, e Human neutrophils pretreated with 1, 3, and $10 \mu \mathrm{M}$ necrosulfonamide (NSA) or 50,100 , and $200 \mu \mathrm{M} \mathrm{Nec}$ for $1 \mathrm{~h}$ at $37^{\circ} \mathrm{C}$ and $5 \% \mathrm{CO}_{2}$, and then were stimulated with bacterial supernatant for $1 \mathrm{~h}$. The release of $\mathrm{LDH}$ by neutrophils was detected $(n=3)$. $\mathbf{d}$, $\mathbf{f}$ Levels of $\mathrm{PMLKL}, \mathrm{MLKL}$, and $\beta$-actin in neutrophil lysate in (c) or (e) treatment were detected by western blot. Data were shown as mean $\pm \mathrm{SE}(\mathbf{c}, \mathbf{e}) .{ }^{*} P<0.05,{ }^{* * *} P<0.0001$ vs. supernatant treated group; ${ }^{\# \# \#} P<$ 0.0001 vs. PBS-treated group

inhibitor NSA or RIPK1 inhibitor Nec, significantly reversed the neutrophil lysis caused by PSM $\alpha 1$ (Fig. 5d, e). Moreover, culture supernatants harvested from the agr or psma mutant decreased the release of $\mathrm{LDH}$ and the expression level of pMLKL in neutrophils compared with LAC group (Fig. 5f, g).

\section{PSMa1-induced neutrophil necroptosis was blocked by WRW4 or anti-TNFa}

To figure out whether or not TNF $\alpha$ participates in PSM $\alpha 1$-induced necroptosis in neutrophil cells, the cells were pretreated with TNF $\alpha$ antibody (anti-TNF $\alpha$ ) and subsequently stimulated with PSM $\alpha 1$. Our data showed that addition of an increasing amount neutralizing antiTNF $\alpha$ to the culture medium inhibited obviously PSM $\alpha 1-$ induced LDH release and MLKL phosphorylation (Fig. 6a, c). Consistently, blocking formylpeptide receptor 2 (FPR2) by WRW4 also markedly prevented PSM $\alpha 1$-induced neutrophil necroptosis and decreased the level of TNF $\alpha$ in cell culture (Fig. 6b, d, e) in a concentration-dependent manner.

Inhibiting necroptotic cell death protects the infected mice

A pneumonia mouse model was established using wildtype LAC or $\Delta p s m \alpha$. LAC-infected mice were intraperitoneally injected with $20 \mathrm{mg} / \mathrm{kg}$ RIP or $15 \mathrm{mg} / \mathrm{kg} \mathrm{Nec}$. LAC-infected mice treated with RIP, Nec, or mice infected by $\Delta p s m \alpha$ strain achieved higher survival rates relative to those of the LAC-infected group (Fig. 7a). The number of $S$. aureus colony-forming units (CFU) in the lung tissue of the LAC-infected mice was significantly higher than that after RIP, Nec, or $\Delta p s m \alpha$ treatment (Fig. 7b). HE data showed that the LAC-infected mice presented with less lung injury after RIP and Nec administration or $\Delta p s m \alpha$ infection than before these treatments were given (Fig. 7c). Flow cytometry data revealed that the percentage of necrotic neutrophils in the lung tissue of the LAC-infected mice increased to 


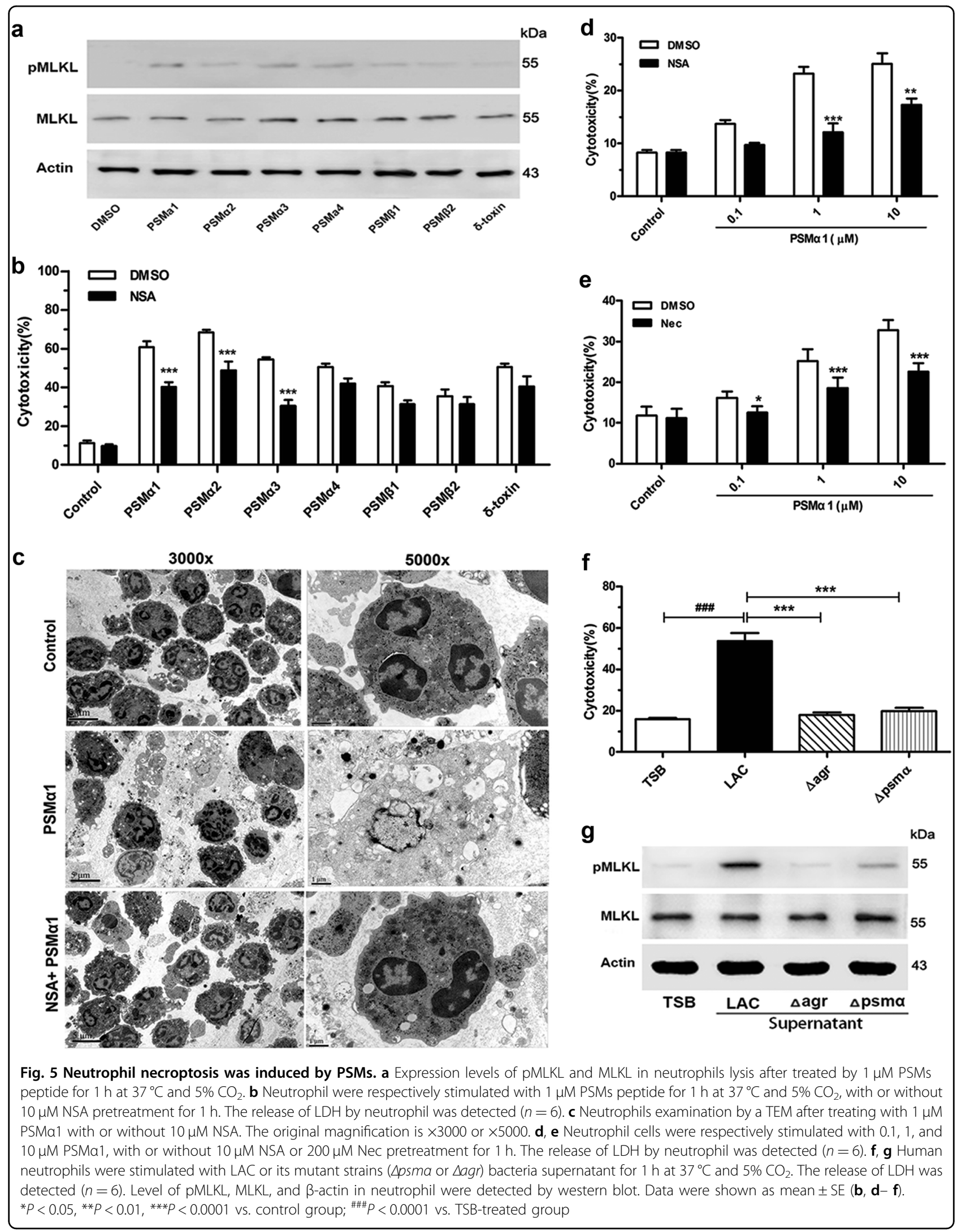



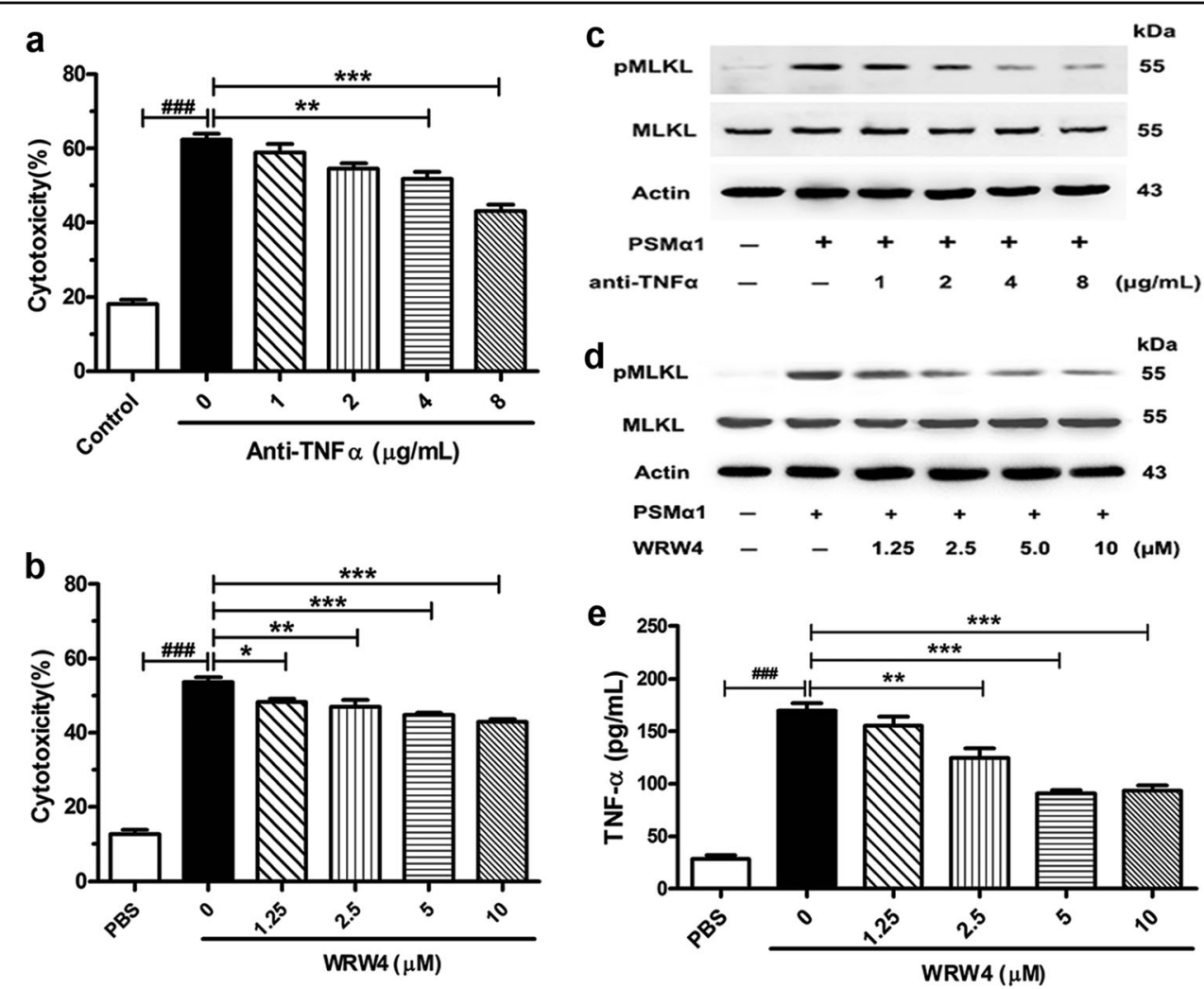

Fig. 6 PSMa1-induced neutrophil necroptosis was blocked by WRW4 or anti-TNFa. a, c Neutrophil were treated with PBS or TNFa neutralizing antibody at indicated concentrations for $1 \mathrm{~h}$ prior to the incubation with $1 \mu \mathrm{M}$ PSMa 1 for $1 \mathrm{~h}$. The release of LDH was detected $(n=6)$ and the level of pMLKL, MLKL and $\beta$-actin in neutrophil were detected by western blot $(n=3)$. $\mathbf{b}, \mathbf{d}$, e Neutrophil were treated with PBS or WRW4 at indicated concentrations for $1 \mathrm{~h}$ prior to the incubation with $1 \mu \mathrm{M}$ PSMa1 for $1 \mathrm{~h}$. The release of LDH was detected $(n=6)$, the level of pMLKL, MLKL and $\beta$ actin in neutrophil were detected by western blot $(n=3)$, and the level of TNFa was detected by ELISA assay $(n=8)$. Data were shown as mean \pm SE $(\mathrm{a}, \mathrm{b}, \mathrm{e}) .{ }^{*} P<0.05,{ }^{* *} P<0.01,{ }^{* *} P<0.001$ vs. control group; ${ }^{\# \# \#} P<0.0001$ vs. PBS-treated group

$56.6 \%$, but became $28.3 \%, 31.3 \%$, or $41.9 \%$ after $\Delta p s m \alpha$, RIP, or Nec treatment, respectively (Fig. 7d). Immunofluorescence results showed that the necroptotic neutrophils were less in the lung tissue than in the model mice after the LAC-infected mice were injected with RIP or Nec or infected with $\Delta p s m \alpha$ (Fig. 7e). Western blot analysis revealed that the neutrophils in the LAC-infected mice decreased in pMLKL level after $\Delta p s m \alpha$, Nec, or RIP treatment (Fig. 7f).

\section{Discussion}

The widespread use of common antimicrobial agents against MRSA has triggered a surge in multidrug-resistant $S$. aureus. The cost of treating MRSA infections is higher than that of methicillin-susceptible $S$. aureus and inflicts tremendous economic and medical burden on patients and the healthcare system. Anti-virulence therapy is a promising strategy that targets $S$. aureus pathogenicity rather than viability. As such, this method controls the severity of an infectious disease while bypassing the evolutionary pressure on the bacterium to develop resistance.
RIP, as an agr quorum sensing system in staphylococcal strains, inhibits the expression of many virulence factors and has exerted individual or synergistic protective effects in several mouse sepsis models ${ }^{20}$, 21 . In the present study, the protective effects of RIP were confirmed in a pneumonia infection model. After RIP treatment in vivo, the bacterial burden was reduced significantly in the lung and BALF of infected mice (Fig. 1). Moreover, the pathological damage to the infected organs was alleviated, and the general weight and survival rate were improved significantly (Fig. 1).

Most PSMs are regulated tightly by the agr system. The core peptide sequence of PSMs is highly conserved in $S$. aureus strains. PSMs are categorized as $\alpha$-type and $\beta$-type. The promoters of $p s m \alpha$ and $p \sin \beta$ genes have been confirmed to be directly regulated by $\operatorname{AgrA}^{22}$. PSM $\alpha$ is a toxin associated with severe necrotizing pneumonia ${ }^{23,}{ }^{24}$. PSM $\alpha$ is produced by most staphylococci and released at high levels by CA-MRSA ${ }^{12,13}$. Studies have shown that HAMRSA strains that overexpress the psm $\alpha$ genes more readily cause infection than that with the regular express 


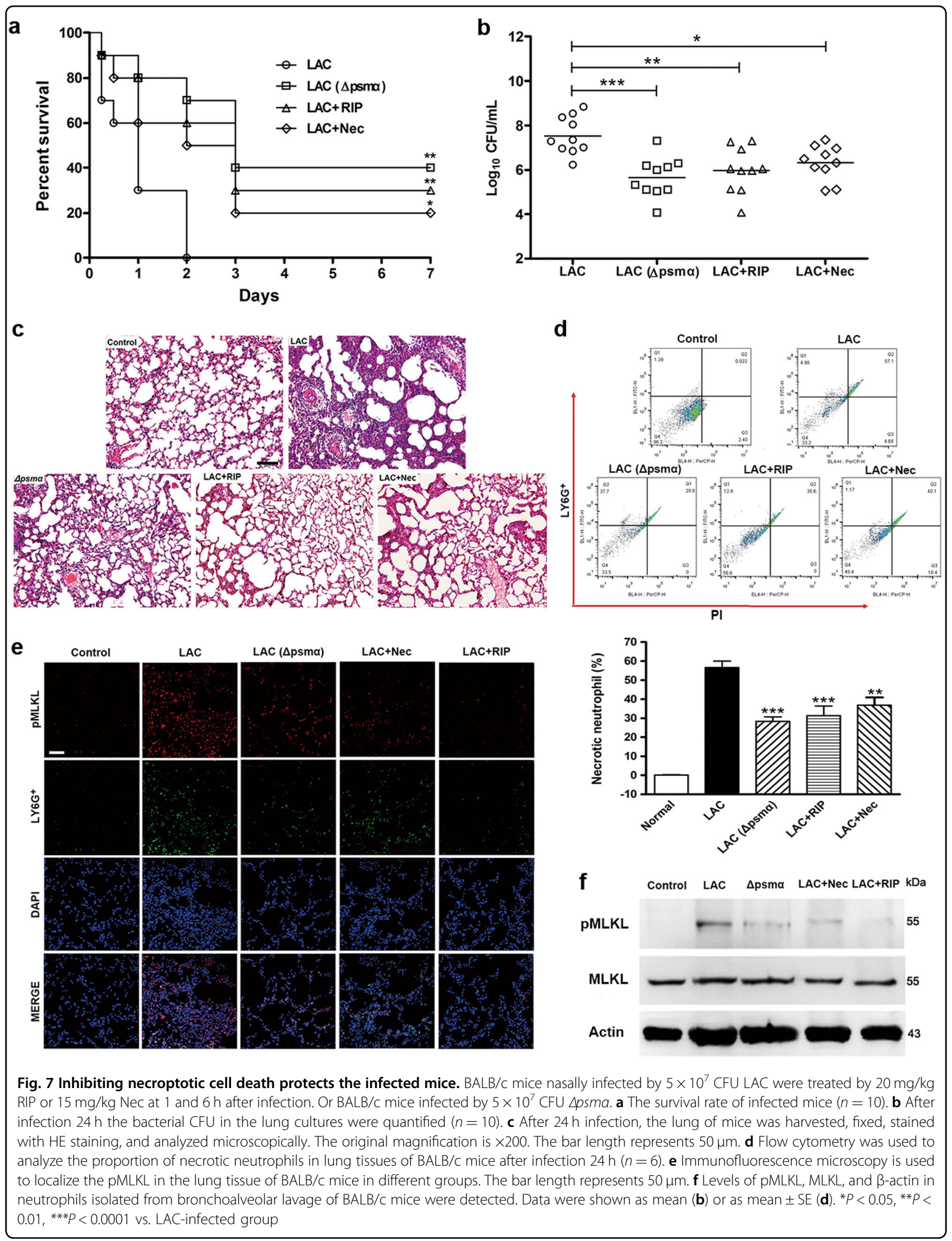


of the psma genes ${ }^{14,15}$. Meanwhile, the $\beta$-type PSMs of Staphylococcus epidermidis play a key role in biofilm development ${ }^{25}$. All of these genes are tightly regulated by $a g r$ and are particularly important in the $S$. aureus pathogenesis. Thus, the expression and virulence of these genes were investigated. The agrA expression was markedly decreased by RIP in a concentration-dependent manner (Fig. 2). Moreover, agrA blockade significantly inhibited the expression of agrA-regulated virulence genes, including $p s m \alpha$ and $p s m \beta$, in S. aureus (Fig. 2).

Neutrophils and macrophages are the two most important cellular defenses against invading $S$. aureus. Their phagocytosis is a highly effective effect for $S$. aureus clearance $^{26-28}$. Moreover, RIP exhibits no antibacterial activity in vitro, thus, a neutrophil- or macrophagedeleted mouse infection model was used to investigate which immune cell plays a critical role in the protective effects of RIP in vivo. Our results revealed that relative to normal LAC-infected mice, the LAC-infected mice treated with RIP exerted protective effects even after their macrophages were deleted (Fig. 3). However, the protective effects of RIP completely disappeared in the neutropenic mice. All these data confirmed that the in vivo antibacterial activity of RIP is highly associated with neutrophil function. To exclude the direct effects of RIP on neutrophils, we detected the MPO activity, cell viability and cytotoxicity of neutrophils under different RIP concentrations. Results indicated that RIP did not affect neutrophil viability and did not induce the release of MPO and LDH (Fig. 3). Therefore, neutrophils play a key role in the anti-infective effects of RIP and RIP may play a protective role in vivo by indirectly affecting neutrophils.

The pathogenicity of LAC is associated with toxin production. S. aureus, especially the CA-MRSA strain, can overcome neutrophil-mediated phagocytosis and succeed in evading destruction by neutrophils. Ultimately, neutrophil lysis ensues ${ }^{29,} 30$. Moreover, the S. aureus surviving inside neutrophils facilitates neutrophil lysis, which then results in the release of cell contents that promote local inflammation ${ }^{29},{ }^{30}$. Understanding the molecular mechanisms by which $S$. aureus avoids neutrophilmediated responses and induces neutrophil lysis may provide insights into the effects of the agr inhibitor in vivo. The survival of $S$. aureus within neutrophils has been reported to undergo programmed necroptosis ${ }^{17}$. We postulated that RIPK1/RIPK3/MLKL-mediated necroptosis is likely involved in LAC-induced cytotoxicity. Our results confirmed that LAC or LAC supernatant can increase the level of pMLKL in human neutrophils, and the effect can be prevented by NSA or Nec (Fig. 4). However, HKLAC did not induce the phosphorylation of MLKL. Meanwhile, NSA or Nec also significantly reduced the supernatant-induced lysis of neutrophils dosedependently. All the above-mentioned data indicated that the $S$. aureus culture supernatant may participate in the neutrophil necroptosis signaling pathway.

Studies have reported that PSMs are cytolytic for neutrophils in the micromolar range ${ }^{11}$. Through fluorescentlabeled S. aureus, Surewaard et al. found that PSM $\alpha$ contributes to neutrophil cell death and raises $S$. aureus survival $^{15}$. Therefore, seven toxins of PSMs were synthesized to investigate whether these PSMs can induce neutrophil necroptosis. Our studies demonstrated that PSM $\alpha 1,2$, and 3 can induce the MLKL phosphorylation and increase neutrophil cytotoxicity significantly (Fig. 5). TEM and cytotoxicity results also showed that NSA, as an MLKL inhibitor, can significantly reverse the neutrophil lysis caused by PSM $\alpha 1$. Moreover, culture supernatants harvested from the agr or psma mutant decreased LDH release and the expression level of pMLKL, which further confirmed the effect of PSM $\alpha$ on inducing neutrophil necroptosis.

TNF $\alpha$ plays an important role in cell necrotic death by RIPK3 signaling pathway ${ }^{31,32}$. TNF $\alpha$ also induces neutrophil necroptosis ${ }^{27}, 28$. To figure out whether or not TNF $\alpha$ participates in PSM $\alpha 1$-induced necroptosis in neutrophil cells, the cells were pretreated with anti-TNF $\alpha$ and subsequently stimulated with PSM $\alpha 1$. Our results showed that PSM $\alpha 1$-induced necroptosis was inhibited concentration-dependently with the increase in antiTNF $\alpha$ as shown in Fig. 6. This finding definitely indicated that PSM $\alpha 1$ induced necroptosis depends on autocrine TNF $\alpha$.

Until now, it's still unclear how PSM $\alpha 1$ induces TNF $\alpha$ secretion in neutrophil. Cytolytic peptides PSM $\alpha$ peptides are more selective for FPR2 than for FPR $1^{33}$. FPR2 is a specific receptor for PSM ${ }^{34,35}$, although most bacteriaderived formyl peptides are more potent at FPR1 than FPR2. Moreover, the virulence regulator agr controls the staphylococcal capacity to activate human neutrophils via the FPR $2^{36}$. Basing on these reports, we speculated that PSM $\alpha 1$ may induce TNF $\alpha$ secretion by FPR 2 in neutrophils. To verify this hypothesis, we pretreated with FPR2 inhibitor WRW4 and then detected the necroptosis of neutrophils induced by PSM $\alpha 1$. Our data showed that blocking FPR2 by WRW4 also markedly prevented PSM $\alpha 1$-induced neutrophil necroptosis and decreased the level of TNF $\alpha$ in cell culture (Fig. 6) in a concentration dependent manner. All these results indicated that PSM $\alpha 1$ induced TNF $\alpha$ secretion through FPR2 and autocrine TNFa subsequently caused neutrophil necroptosis.

Interfering with the bacterial virulence release pathways is a potential approach because of the ability of this strategy to bypass evolutionary pressure on the bacterium to develop resistance. This ability is absent in traditional bactericidal strategies ${ }^{8,9,37}$. Our data firstly indicated $S$. aureus-secreted PSM $\alpha$ can induce neutrophil necroptosis 
by promoting TNF $\alpha$ autocrine and exacerbate severe tissue damage. Moreover, blocking agrA and psma expression significantly suppressed the neutrophil necroptosis induced by $S$. aureus and effectively prevented pneumonia through the $p s m \alpha$ mutant strain, with RIP as agr inhibitor and Nec as RIPK1 inhibitor. Hence, the blockage of the agr system is a promising strategy for inhibiting the neutrophil necroptosis of infected mice and improving the clearance of S. aureus from infected mice.

\section{Materials and methods}

\section{Bacteria and agents}

S. aureus ATCC29213 and S. epidermidis ATCC14990 were obtained from the Chinese National Center for Surveillance of Antimicrobial Resistance. Mu50 ATCC700699 was purchased from MicroBiologics (Minnesota, USA). LAC (USA 300) and its mutant strains ( $\triangle p s m \alpha$ and $\triangle a g r$ ) were generous gifts from Michael Otto (National Institute of Allergy and Infectious Diseases, MD). MRSA XJ75302 and methicillin-resistant S. epidermidis (MRSE) XJ75284 were obtained from the clinical laboratory of Xijing Hospital (Xi'an, China). The S. aureus strains used are listed in Supplementary Table 2.

Vancomycin, oxacillin, ciprofloxacin, ceftazidine, and levofloxacin were purchased from the National Institute for the Control of Pharmaceutical and Biological Products (Beijing, China). RIP $\left(\mathrm{CH}_{3} \mathrm{CO}-\mathrm{YKPVTNF}-\mathrm{CONH}_{2}\right)$ and PSMs (Supplementary Table 1) were synthesized and the purity of the peptide is $>95 \%$. NSA (Calbiochem, USA) is a pharmacological inhibitor of MLKL. Nec (Calbiochem, USA) is an inhibitor of RIPK1. WRW4 (Alomone Labs, USA) is an antagonist of FPR2.

\section{Antimicrobial susceptibility and growth assay}

Minimal inhibitory concentrations (MICs) of peptides and antibiotic were performed in sterile, flat-bottomed 96-well microplates according to the broth microdilution guidelines of Clinical and Laboratory Standards Institute $(\text { CLSI })^{38}$.

To determine the growth curve for bacteria, the working suspension of the inoculum $\left(2 \times 10^{7} \mathrm{CFU} / \mathrm{mL}, 160 \mu \mathrm{L}\right)$ was cultivated in the automated Bioscreen $\mathrm{C}$ system (Lab systems Helsinki, Finland), using a $\mathrm{MH}$ broth culture medium. And $40 \mu \mathrm{L}$ synthetic peptide solution was added to strain cultures to a final concentration of 250,500 , or $1000 \mu \mathrm{g} / \mathrm{mL}$. The optical density of the cell suspensions was measured at $630 \mathrm{~nm}$ in regular intervals of $1 \mathrm{~h}$ for 22 h.

\section{Pneumonia model and BALF collection}

$\mathrm{BABL} / \mathrm{c}$ mice weighing $\sim 20 \mathrm{~g}$ were housed for 7 days prior to inoculation. Mice were anesthetized intraperitoneal injection with $400 \mathrm{mg} / \mathrm{kg}$ chloral hydrate and hung in an upright position, and inoculated with LAC $\left(3 \times 10^{7}\right.$
$\mathrm{CFU}$ ) in a volume of $30 \mu \mathrm{L}$ PBS into the right nares. Keep the infectious mice in the upright position for $1 \mathrm{~min}$. Control mice received $30 \mu \mathrm{L}$ sterile PBS. Treatment was initiated at 1 and $6 \mathrm{~h}$ after infection. To observe the weight recovery, the mice were weighed at $0,1,2,3,5$, and 7 days (at a fixed time) after infection. Mice were monitored every $6 \mathrm{~h}$ after infection, and survivors were euthanized for 7 days. Lungs were harvested at $24 \mathrm{~h}$ after infection. The right lung was homogenized in $1 \mathrm{~mL}$ of PBS, and serial dilutions were plated in duplicate on agar plates for evaluating bacterial burden in the infected organs. The left lung of infected mice were inflated with $10 \%$ buffered formalin, processed, stained with HE stain, and analyzed microscopically. All HE-stained sections were scored by a pathologist who was blinded to study-group attribution. The total pathologic score for each mouse was calculated as the sum of scores from each category for that individual.

Pneumonia mice were anesthetized with an intraperitoneal injection of chloral hydrate $(400 \mathrm{mg} / \mathrm{kg})$. The trachea was cannulated (22 GA Insyte, Becton Dickinson), and $1 \mathrm{~mL}$ cold PBS was infused intratracheally and withdrawn. This procedure was repeated three times, resulting in a total volume of $2.5 \mathrm{~mL}$. The bacterial CFU in BALF were quantified by plating serial dilutions on $\mathrm{MH}$ agar plates and enumerating colonies. To quantify the degree of pulmonary edema, we detected the lung water content as described previously. The lung weight was measured immediately after its excision (wet weight). The lung tissue was then dried in an oven at $60^{\circ} \mathrm{C}$ for $72 \mathrm{~h}$. Lung wet/ dry weight $(\mathrm{W} / \mathrm{D})$ ratio $=$ wet weight/dry weight.

\section{Quantitative RT-PCR}

The in vivo agrA and psma expression levels were measured by directly extracting the MRSA RNA from the lung BALF of infected BALB/c mice ${ }^{39}$. Total RNA was isolated with Trizol and reverse transcription was performed with reverse transcriptase according to the manufacturer's instructions. RT-PCR was performed using multiple kits (SYBR Premix Ex TAQ, Takara Bio, Japan) according to the manufacturer's instructions. To determine the relative expression level of mRNA, each gene was normalized to the expression level of the housekeeping gene 16SrRNA. The primers used in the present study are listed in Supplementary Table 3.

\section{Macrophage or neutrophil depletion in mice}

Macrophages were depleted by a single tail vein administering $150 \mu \mathrm{L}$ clodronate liposomes (Clodronate Liposomes. org, Netherlands). Then mice were challenged with LAC $\left(3 \times 10^{7} \mathrm{CFU}\right)$ by intraperitoneal injection. Infected-mice were sacrificed at $0,24,48,96$, and $120 \mathrm{~h}$ later. Macrophage depletion was confirmed by flow cytometry in peritoneal lavage fluid. 
Mice were rendered neutropenic by a single tail vein injection cyclophosphamide (CTX, $200 \mathrm{mg} / \mathrm{kg}$ ). Peritoneal lavage fluid was collected and the percentage of macrophage was detected by flow cytometry after the mice were intraperitoneal administered with LAC $\left(3 \times 10^{7} \mathrm{CFU}\right)$. Blood was collected from the retro-orbital sinuses of anesthetized to determine the extent of neutropenia at 0 , $24,48,96$, and $120 \mathrm{~h}$ after injection. The blood was analyzed with an Abbott Cell-Dyn 3700 system.

\section{Neutrophil isolation and co-culture with S. aureus, supernatant, or PSM}

Human neutrophils were isolated from the blood of healthy volunteers by standard Ficoll/Histopaque gradient centrifugation. The resulting preparation was found to be over 98\% neutrophils according to Wright-Giemsa staining. Trypan blue staining to identify cell viability was over 95\%. Neutrophil cells were grown in RPMI Medium 1640 (Gibco) with $10 \%$ fetal bovine serum. Human neutrophils were challenged with LAC at multiplicities of infection (MOI 10:1; CFU of $S$. aureus: neutrophils). Neutrophil cells were pretreated $1 \mathrm{~h}$ with NSA $(1,3$ or $10 \mu \mathrm{M})$ or Nec $(50,100$ or $200 \mu \mathrm{M})$ and then were stimulated with LAC (WT, $\Delta p s m \alpha, \Delta a g r$ ) supernatant or Heat-killed LAC (HKLAC) for $1 \mathrm{~h}$ at $37^{\circ} \mathrm{C}$ and $5 \% \mathrm{CO}_{2}$.

\section{MPO activity}

Neutrophils $\left(1 \times 10^{6}\right)$ was treated with $10,30,100,300$, or $1000 \mu \mathrm{g} / \mathrm{mL}$ RIP or $50 \mathrm{ng} / \mathrm{mL}$ LPS at $37^{\circ} \mathrm{C}$ in $5 \% \mathrm{CO}_{2}$ for $30 \mathrm{~min}$. Cells were collected by centrifugation at 1000 rpm for $5 \mathrm{~min}$ and were lysed to getting cell-free supernatant for MPO activity. MPO activity of neutrophil was measured by spectrophotometer according to the instructions of MPO assay kit.

\section{Human TNFa enzyme-linked immunosorbent assay}

Neutrophil cells were stimulated with $1 \mu \mathrm{M}$ PSM $\alpha 1$ at $37^{\circ} \mathrm{C}$ for $1 \mathrm{~h}$ after pretreated with $1.25,2.5,5$, or $10 \mu \mathrm{M}$ WRW4 for $1 \mathrm{~h}$. Then the level of human TNFo in the cell culture supernatant was assessed using an enzyme-linked immunosorbent (ELISA) kit (Boster Biological Technology, China) after centrifugation (1000 rpm, $10 \mathrm{~min}$ ).

\section{Cytotoxicity detection}

Neutrophils was treated with $10,30,100,300$, or 1000 $\mu \mathrm{g} / \mathrm{mL}$ RIP at $37^{\circ} \mathrm{C}$ in $5 \% \mathrm{CO}_{2}$. Cell viability was assessed 24h later using Cell counting Kit 8 (CCK-8, Dojingdo Laboratories, Japan) according to the manufacturer's protocol. The cell-free supernatant is used to detect the release of lactate dehydrogenase (LDH) according to the manufacturer's protocol (Cytotoxicity Detection Kit, Roche).

Neutrophil cells were respectively stimulated with PSM peptide for $1 \mathrm{~h}$ at $37^{\circ} \mathrm{C}$ and then centrifugated
$(1000 \mathrm{rpm}, 10 \mathrm{~min})$ after pretreated with or without NSA, Nec, WRW4, anti-TNF $\alpha$ for $1 \mathrm{~h}$. The neutrophil cell culture supernatant is used to detect the release of LDH.

\section{Flow cytometry analysis}

Mice were sacrificed by cervical dislocation. The peritoneal cavity was lavaged with $3 \mathrm{~mL}$ of ice-cold sterile PBS. The buffer containing resident peritoneal cells was slowly withdrawn. Cells collected by concentration were suspended in fluorescence-activated cell sorter buffer ( $10 \%$ fetal bovine serum and $0.1 \%$ sodium azide in PBS) and stained for $30 \mathrm{~min}$ at $4{ }^{\circ} \mathrm{C}$. The macrophages were stained with the F4/80-FITC macrophage specific glycoprotein. The percentage of $\mathrm{F} 4 / 80$-positive cells was determined by counting cell numbers.

Lung sections were incubated in $2.5 \mathrm{mg} / \mathrm{mL}$ collagenase with $0.5 \mathrm{mg} / \mathrm{mL}$ DNase-1 (Sigma-Aldrich) at $37^{\circ} \mathrm{C}$ for 60 min and red blood cells were lysed with ammonium chloride potassium containing lysing buffer (Gibco) to aquire single-cell suspensions. Cells were suspended in fluorescence-activated cell sorter buffer and stained for $30 \mathrm{~min}$ at $4{ }^{\circ} \mathrm{C}$. Combinations of fluorescein isothiocyanate-labelled (FITC) anti-Ly-6G (Gr-1; RB68C5; Biolegend) and propidium iodide (PI) were used. Macrophages $\left(\mathrm{F} 4 / 80^{+}\right)$and neutrophils (neutrophils; Ly6G ${ }^{+}$) were enumerated using FlowJo V10.

\section{Western blot analysis}

$S$. aureus supernatant was obtained by centrifugation and filtration. HKLAC was obtained by incubating LAC cells in PBS $\left(1 \times 10^{9} \mathrm{CFU} / \mathrm{mL}\right)$ at $65^{\circ} \mathrm{C}$ for $2 \mathrm{~h}$ to inactivate the bacteria. Neutrophil lysates were run on bolt $4-12 \%$ Bis-Tris Plus gels (Life Technologies) and transferred to polyvinylidene difluoride membranes (Millipore). Then the membrane was blocked with $5 \%$ milk in TBST (Trisbuffered saline plus Tween) for $1.5 \mathrm{~h}$ at room temperature. Immunodetection was performed using antiphospho-MLKL (Ser358) (Abcam, Cambridge, UK), anti-MLKL (Abcam, Cambridge, UK), anti-TNF $($ R\&D Systems, USA), and $\beta$-actin (Sigma Aldrich Chemical Co, USA) antibodies followed by secondary antibodies conjugated to horseradish peroxidase (Santa Cruz Biotechnology Inc, USA).

\section{Transmission electron microscopy}

The cells were fixed with ice-cold $2.5 \%$ glutaraldehyde in PBS (pH 7.3) at $4{ }^{\circ} \mathrm{C}$ for $4 \mathrm{~h}$. Fixed cells were post-fixed in $2 \% \mathrm{OsO}_{4}$, dehydrated in graded alcohol, embedded in Epon 812 (Electron Microscopy Sciences, Fort Washington, PA, USA), sectioned with ultramicrotome, and stained with uranyl acetate and lead citrate. Images were acquired under a laser confocal microscope (FV1000, Olympus). 


\section{Statistical analysis}

Results are expressed as mean (Figs. 1c, d, f and 7b), mean \pm standard deviations (Figs. 1e and $3 \mathrm{~b}, \mathrm{~d}$ ), or mean \pm standard error of the mean (Figs. 1a, 2, 3f-i, 4c, e, 5b, d, $6 \mathrm{a}, \mathrm{b}$, e and $7 \mathrm{~d}$ ). Two-way analysis of variance (ANOVA) was used for the data presented in Figs. 1a, 2c, d, 3b, d, f, and $5 \mathrm{~b}, \mathrm{~d}$, e. Comparisons of three or more groups were performed using one-way ANOVA (Figs. 1c-f, 2a, b, 3g-i, 4c, e, 5f, 6a, b, e and 7b, d). Survival curves were calculated by the Kaplan-Meier method (Figs. 1b, 3a, c, e and 7a). Statistical analyses were done using Prism software (version 6; GraphPad, CA). A probability value of $P<0.05$ was considered statistically significant.

\section{Ethics statement}

Animal experiments were carried out in strict accordance with National Institutes of Health guidelines for the care and use of laboratory animals and approved by the Institutional Animal Care and Use Committee of the Fourth Military Medical University. The present study was approved by the Medical Ethical Committees of the Fourth Military Medical University (XJYYLL-2014489).

\section{Acknowledgements}

We thank Michael Otto for providing us with LAC and its mutant strains (psma and agr mutant). This work was supported by grants from the National Natural Science Foundation of China (no. 81673477, 81471997, 81402931, 81473252, and 81001460), the State Project for Essential Drug Research and Development (2013ZX09J13108), and the Innovation plan of science and technology of Shaanxi Province (2013KTCQ03-04).

\section{Author details}

${ }^{1}$ Department of Pharmacology, School of Pharmacy, Fourth Military Medical University, Xi'an, China. ${ }^{2}$ Department of Pharmacology, Xi'an Medical University, Xi'an, China. ${ }^{3}$ Henan Eye Institute, Henan Provincial People's Hospital, Zhengzhou, China. ${ }^{4}$ Department of Clinical Laboratory Medicine, Xijing Hospital, Fourth Military Medical University, Xi'an, China

\section{Conflict of interest}

The authors declare that they have no conflict of interest.

\section{Publisher's note}

Springer Nature remains neutral with regard to jurisdictional claims in published maps and institutional affiliations.

Supplementary Information accompanies this paper at (https://doi.org/ 10.1038/s41419-018-0398-z).

Received: 1 November 2017 Revised: 6 February 2018 Accepted: 12 February 2018

Published online: 02 March 2018

\section{References}

1. Khokhlova, O. E. et al. Healthcare- and community-associated methicillinresistant Staphylococcus aureus (MRSA) and fatal pneumonia with pediatric deaths in Krasnoyarsk, Siberian Russia: unique MRSA's multiple virulence factors, genome, and stepwise evolution. PLOS ONE 10, e0128017 (2015).

2. Takada, H. et al. Linezolid versus vancomycin for nosocomial pneumonia due to methicillin-resistant Staphylococcus aureus in the elderly: a retrospective cohort analysis: effectiveness of linezolid in the elderly. Am. J. Emerg. Med. 35, 245-248 (2017)

3. Lee, J. Y. \& Howden, B. P. Vancomycin in the treatment of methicillin-resistant Staphylococcus aureus - a clinician's guide to the science informing current practice. Expert Rev. Anti Infect. Ther. 13, 855-869 (2015).

4. Pomorska-Wesolowska, $M$. et al. Virulence and antimicrobial resistance of Staphylococcus aureus isolated from bloodstream infections and pneumonia in Southern Poland. J. Glob. Antimicrob. Resist. 11, 100-104 (2017).

5. Shariq, A. et al. Susceptibility profile of methicillin-resistant Staphylococcus aureus to linezolid in clinical isolates. Int. J. Health Sci. 11, 1-4 (2017).

6. Menif, K. et al. Community-associated methicillin-resistant Staphylococcus aureus infections in a pediatric intensive care unit. J. Infect. Dev. Ctries. 5, 587-591 (2011).

7. Rutherford, S. T. \& Bassler, B. L. Bacterial quorum sensing: its role in virulence and possibilities for its control. Cold Spring Harb. Perspect. Med 2, a012427 (2012).

8. Singh, R. P., Desouky, S. E. \& Nakayama, J. Quorum quenching strategy targeting Gram-positive pathogenic bacteria. Adv. Exp. Med. Biol. 901, 109-130 (2016).

9. Kalia, V. C. \& Purohit, H. J. Quenching the quorum sensing system: potential antibacterial drug targets. Crit. Rev. Microbiol. 37, 121-140 (2011).

10. Gov, Y., Bitler, A., Dell'Acqua, G., Torres, J. V. \& Balaban, N. RNAlll inhibiting peptide (RIP), a global inhibitor of Staphylococcus aureus pathogenesis: structure and function analysis. Peptides 22, 1609-1620 (2001).

11. Peschel, A. \& Otto, M. Phenol-soluble modulins and staphylococcal infection. Nat. Rev. Microbiol. 11, 667-673 (2013).

12. Otto, M. Phenol-soluble modulins. Int. J. Med. Microbiol. 304, 164-169 (2014).

13. Wang, R. et al. Identification of novel cytolytic peptides as key virulence determinants for community-associated MRSA. Nat. Med. 13, 1510-1514 (2007).

14. Diep, B. A. \& Otto, M. The role of virulence determinants in communityassociated MRSA pathogenesis. Trends Microbiol. 16, 361-369 (2008).

15. Surewaard, B. G. et al. Staphylococcal alpha-phenol soluble modulins contribute to neutrophil lysis after phagocytosis. Cell. Microbiol. 15, 1427-1437 (2013).

16. Cheung, G. Y., Joo, H. S., Chatterjee, S. S. \& Otto, M. Phenol-soluble modulins-critical determinants of staphylococcal virulence. FEMS Microbiol. Rev. 38, 698-719 (2014).

17. Greenlee-Wacker, M. C. et al. Phagocytosis of Staphylococcus aureus by human neutrophils prevents macrophage efferocytosis and induces programmed necrosis. J. Immunol. 192, 4709-4717 (2014).

18. Orozco, S. \& Oberst, A. RIPK3 in cell death and inflammation: the good, the bad, and the ugly. Immunol. Rev. 277, 102-112 (2017).

19. Chan, F. K., Luz, N. F. \& Moriwaki, K. Programmed necrosis in the cross talk of cell death and inflammation. Annu. Rev. Immunol. 33, 79-106 (2015).

20. Ghiselli, R. et al. RNAll-inhibiting peptide in combination with the cathelicidin BMAP-28 reduces lethality in mouse models of staphylococcal sepsis. Shock 26, 296-301 (2006).

21. Giacometti, A. et al. RNAlll-inhibiting peptide improves efficacy of clinically used antibiotics in a murine model of staphylococcal sepsis. Peptides 26, 169-175 (2005).

22. Queck, S. Y. et al. RNAlll-independent target gene control by the agr quorumsensing system: insight into the evolution of virulence regulation in Staphylococcus aureus. Mol. Cell 32, 150-158 (2008).

23. Berube, B. J., Sampedro, G. R., Otto, M. \& Bubeck Wardenburg, J. The psmalpha locus regulates production of Staphylococcus aureus alpha-toxin during infection. Infect. Immun. 82, 3350-3358 (2014).

24. Bonesso, M. F. et al. Key role of alpha-toxin in fatal pneumonia caused by Staphylococcus aureus sequence type 398. Am. J. Respir. Crit. Care Med. 193, 217-220 (2016).

25. Otto, M. Staphylococcal biofilms. Curr. Top. Microbiol. Immunol. 322, 207-228 (2008).

26. McGuinness, W. A., Kobayashi, S. D. \& DeLeo, F. R. Evasion of neutrophil killing by Staphylococcus aureus. Pathogens 5, 32 (2016).

27. Zhao, H. et al. Role of necroptosis in the pathogenesis of solid organ injury. Cell Death Dis. 6, e1975 (2015).

28. Wicki, S. et al. Loss of XIAP facilitates switch to TNFalpha-induced necroptosis in mouse neutrophils. Cell Death Dis. 7, e2422 (2016).

29. Greenlee-Wacker, M., DeLeo, F. R. \& Nauseef, W. M. How methicillin-resistant Staphylococcus aureus evade neutrophil killing. Curr. Opin. Hematol. 22, 30-35 (2015). 
30. Guerra, F. E., Borgogna, T. R., Patel, D. M., Sward, E. W. \& Voyich, J. M. Epic immune battles of history: neutrophils vs. Staphylococcus aureus. Front. Cell Infect. Microbiol. 7, 286 (2017).

31. He, S. et al. Receptor interacting protein kinase-3 determines cellular necrotic response to TNF-alpha. Cell 137, 1100-1111 (2009).

32. Zhang, D. W. et al. RIP3, an energy metabolism regulator that switches TNFinduced cell death from apoptosis to necrosis. Science 325, 332-336 (2009).

33. Otto, M. Community-associated MRSA: what makes them special? Int. J. Med. Microbiol. 303, 324-330 (2013).

34. Kretschmer, D. et al. Human formyl peptide receptor 2 senses highly pathogenic Staphylococcus aureus. Cell Host Microbe 7, 463-473 (2010).

35. He, H. Q. \& Ye, R. D. The formyl peptide receptors: diversity of ligands and mechanism for recognition. Molecules 22, 455 (2017).
36. Kretschmer, D., Nikola, N., Durr, M., Otto, M. \& Peschel, A. The virulence regulator Agr controls the staphylococcal capacity to activate human neutrophils via the formyl peptide receptor 2. J. Innate Immun. 4, 201-212 (2012)

37. Rasko, D. A. \& Sperandio, V. Anti-virulence strategies to combat bacteriamediated disease. Nat. Rev. Drug. Discov. 9, 117-128 (2010).

38. Ma, B. et al. The disulfide bond of the peptide thanatin is dispensible for its antimicrobial activity in vivo and in vitro. Antimicrob. Agents Chemother. 60, 4283-4289 (2016).

39. Moreira, C. G., Weinshenker, D. \& Sperandio, V. QseC mediates Salmonella enterica serovar typhimurium virulence in vitro and in vivo. Infect. Immun. $\mathbf{7 8}$ 914-926 (2010). 